\title{
A statistical approach of the decay of a soliton in a randomly perturbed Toda chain
}

\author{
Josselin Garnier \\ Laboratoire de Statistique et Probabilits, Universite Paul Sabatier, \\ 118 Route de Narbonne, 31062 Toulouse Cedex 4, France \\ E-mail: 'garnier@cict.fri \\ Fatkhulla Abdullaev* ${ }^{\dagger}$ \\ Instituto de Fisica Teórica, UNESP, Rua Pumplona, 145, 01405-900, Sao Paulo, Brasil \\ E-mail: 'f́atkh@ift.unesp.bri'
}

\begin{abstract}
This paper addresses the soliton dynamics in a Toda lattice with a randomly distributed chain of masses. Applying the inverse scattering transform we derive effective equations for the decay of the soliton amplitude that take into account radiative losses. The decay rate does not depend on the incoming energy for large amplitude soliton. An important feature is the generation of a soliton gas consisting of a large collection of small solitons. The soliton gas plays an important role in that the changes in the conservation equations cannot be correctly understood if the soliton production is neglected.
\end{abstract}

KEYwords: 'discrete solitons, random masses, Inverse Scattering Transform, soliton gasi.

\section{Introduction}

The propagation of nonlinear waves in disordered media was recently the subject of many investigations. The joint action of the disorder and nonlinearity leads to many new effects in wave dynamics $[1, i=2,1]$. One of them is the competition between the Anderson localization effect and the localization effects due to the existence of solitons in nonlinear systems.

In linear systems the disorder typically leads to the localization of the wave - the phenomenon first predicted by Anderson for the electron in the disordered lattice [3īi] characteristic scale is the localization length $L_{A}$, characterizing the decay of wavefunction. Later it was shown that this phenomenon can exists for many type of excitations in quantum

${ }^{*}$ Speaker.

${ }^{\dagger}$ On leave of absence from Physical-Technical Institute, Tashkent, Uzbekistan 


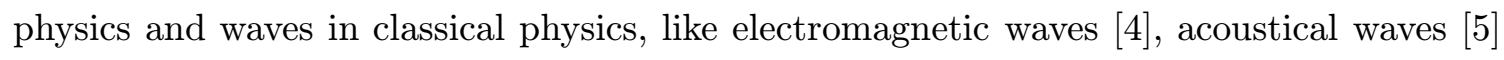
etc.

In nonlinear disordered systems solitons start to play the important role. With solitons the additional scale connects: a nonlinearity length $L_{N L}$. The competition of these scales is responsible for the existence of the mass threshold in envelope solitons propagation in random media. Below this threshold the nonlinear wave decay exponentially, above them solitons lose the mass very slowly [2:-i, $\left.\overline{6_{i}}\right]$.

In nonlinear discrete media numerical simulations shows the existence of power type decay of solitons. Such results has been observed for the nontopological kink-type soliton in random masses anharmonic lattice with nonlinear nearest-neighbor interaction of quartic type - the random Fermi-Pasta-Ulam (FPU) chain [i] in the same type of lattice with disorder in the force constants of harmonic and anharmonic potentials [i] for the transmission coefficient of the leading soliton is $T_{1}=E_{\text {out }} / E_{\text {in }} \sim 1 / \sqrt{N}$ (where $E$ is the soliton energy) has been obtained.

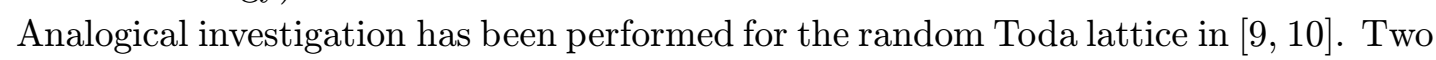
kinds of particles with the different masses were randomly distributed along an impurity segment. For the soliton energy it is found that the dependence of the transmission rate $T_{1}$ can be fitted quite well by $1 /\left(1+\alpha N^{\beta}\right)$, whith $\beta \approx 1.2$ for the wavenumber equal 1 in the dimensionless variables. It is also shown that the decay rate as a function of the wavenumber of the incident soliton can be represented by a Lorentzian function for small wavenumbers and tends to a finite value in the large wavenumber limit.

Theoretical description of these phenomena involves models with a dilute system of impurities, when the distance between impurities are larger then the soliton width and each interaction of soliton with impurity can be considered as the individual one [iin: i2i. Another approach consists in the using of the continuum approximation, and studiyng the stochastically perturbed wave equations. In the work [i] 1 in $]$ in this approach has been applied to the random FPU and decay law for the soliton amplitude $1 / \sqrt{N}$ has been derived. But this consideration, considering only the broad solitons, use indeed the mean field theory and neglect by the radiation phenomena that are important for the long distance propagation in random chain and in generally is questional for the nonlinear waves in random media

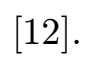

The random extension of the discrete NLS equation type model - the random Ablowitz-

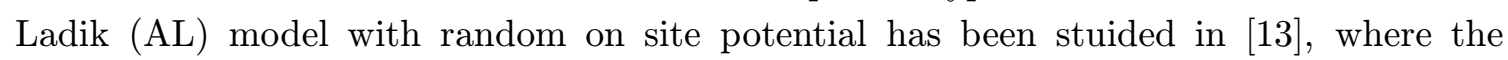
decay law of AL soliton has been found. In this work we will consider the problem of the discrete nontopological kink type soliton propagation in disordered lattice on the example of the random Toda lattice. Few motivations exist for the consideration of this model. First, the Toda model is interesting from the applications point of view and appears in

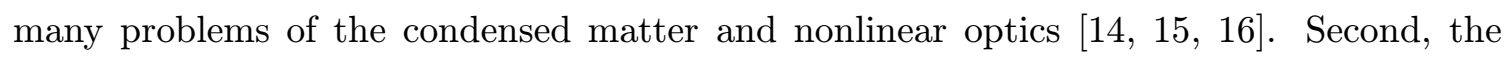
unperturbed Toda model is the integrable system [1] under random modulations of lattice parameters can be studied using for example the perturbation theory based on the Inverse Scattering Transform. Last method admits to calculate the effects of radiation on the propagation of discrete soliton.

In this paper we shall consider the Toda chain with a segment containing random 
masses. The length of the segment of the chain with random masses is assumed to be long $\sim 1 / \varepsilon^{2}$, where $\varepsilon$ is the perturbation amplitude. In comparison with the random AL chain new phenomena are here possible. One of them is the generation of a soliton gas in the Toda chain driven by a random perturbation.

The paper is organized as follows. In the second section we give a review of the homogeneous Toda chain and an introduction to the inverse scattering transform (IST) that is necessary to analyze the long distance evolution of the Toda lattice soliton driven by a random perturbation. In the third section we derive the evolution equations for the scattering data under random perturbations and the equations for the soliton parameters taking into account the radiative losses. We analytically study the decay law of the discrete soliton and we exhibit different regimes depending on the soliton parameter and the correlation length of the medium.

\section{The homogeneous Toda chain}

The model consists of a one-dimensional chain of particles. Each particle with mass 1 interacts through a nearest neighbor exponential potential. The difference equation that governs the dynamics of a one-dimensional lattice with exponential interaction of nearest neighbors is deduced from Newton's law [i]1핌] :

$$
\ddot{x}_{n}=\exp \left(x_{n+1}-x_{n}\right)-\exp \left(x_{n}-x_{n-1}\right),
$$

where $x_{n}$ is the longitudinal displacement of the $n$-th particle from its equilibrium position. The Hamiltonian of this system is:

$$
\mathcal{H}_{0}=\sum_{n=-\infty}^{\infty} \frac{1}{2} \dot{x}_{n}^{2}+\left(\exp \left(x_{n}-x_{n-1}\right)-\left(x_{n}-x_{n-1}\right)-1\right) .
$$

In this section we give a review and extend the main results reported in [i] $\left.\underline{7}_{1}\right]$.

\subsection{Direct scattering transform}

Equation $(2 . \overline{2} \cdot \overline{1})$ can be rewritten as:

$$
\dot{c}_{n}=c_{n}\left(v_{n}-v_{n-1}\right), \quad \dot{v}_{n}=c_{n+1}-c_{n},
$$

where $c_{n}=\exp \left(x_{n}-x_{n-1}\right)$ and $v_{n}=\dot{x}_{n}$. The eigenvalue problem for the continuous spectrum filling the interval $-2 \leq \lambda \leq 2$ reads:

$$
\sqrt{c_{n+1}} f_{n+1}(k)+\sqrt{c_{n}} f_{n-1}(k)+v_{n} f_{n}(k)=\lambda f_{n}(k), \quad \lambda=k+k^{-1},
$$

where $k$ is the spectral parameter that lies in the unit circle $\mathbb{S}^{1}:=\{k \in \mathbb{C},|k|=1\}$. The Jost functions $\psi$ and $\phi$ are the eigenfunctions that satisfy the boundary conditions:

$$
\psi_{n}(k) \stackrel{n \rightarrow \infty}{\simeq} k^{n}, \quad \phi_{n}(k) \stackrel{n \rightarrow-\infty}{\simeq} k^{-n} .
$$

The Jost coefficients are connected to the Jost functions through the identities:

$$
\phi_{n}(k)=a(k) \psi_{n}^{*}(k)+b(k) \psi_{n}(k), \quad \psi_{n}(k)=a(k) \phi_{n}^{*}(k)-b^{*}(k) \phi_{n}(k) .
$$


The Wronskian of two functions $f$ and $g$ is defined by:

$$
W(f, g):=\sqrt{c_{n}}\left(f_{n} g_{n-1}-f_{n-1} g_{n}\right) .
$$

Calculating the Wronskian of $\phi$ and $\psi$ yields:

$$
W(\phi, \psi)=a(k) W\left(\psi^{*}, \psi\right)=a(k)\left(k^{-1}-k\right) .
$$

Another important point as we shall see in the following is that $a$ admits an analytic continuation inside the unit disk. Finally symmetry identities hold true:

$$
a^{*}(k)=a(1 / k), \quad b^{*}(k)=b(1 / k), \quad|a(k)|^{2}-|b(k)|^{2}=1 .
$$

The points on the real axis $k_{r}, r=1, \ldots, R,\left|k_{r}\right|<1$, at which $a\left(k_{r}\right)=0$ correspond one-to-one with eigenvalues of the discrete spectrum. At these points we have:

$$
\phi_{n}\left(k_{r}\right)=b_{r} \psi_{n}\left(k_{r}\right), \quad \operatorname{Im}\left(b_{r}\right)=0 .
$$

Setting $\rho_{r}=b_{r} / a^{\prime}\left(k_{r}\right)$, the set of scattering data $\left\{a(k), b(k), k \in \mathbb{S}^{1}, k_{r}, \rho_{r}, r=1, \ldots, R\right\}$ is sufficient to reconstruct the Jost functions and the function $\left(x_{n}\right)$.

\subsection{Inverse scattering transform}

Given the set of scattering data, set:

$$
\Omega(n)=-\sum_{r=1}^{R} \rho_{r} k_{r}^{n}+\frac{1}{2 i \pi} \oint_{\gamma_{u}} \frac{b(k)}{a(k)} k^{n} d k,
$$

where $\gamma_{u}$ is the positively oriented unit circle. The inverse scattering transform consists in solving the Gel'fand-Levitan-Marchenko (GLM) equation for the kernel $K$ :

$$
K(n, m)+\Omega(n+m)+\sum_{l=n}^{\infty} \Omega(l+m+1) K(n, l)=0 .
$$

Then the function $c_{n}$ and $v_{n}$ are given by:

$$
c_{n}=\frac{1-K(n-1, n-2)}{1-K(n, n-1)}, \quad v_{n}=K(n, n)-K(n-1, n-1) .
$$

\subsection{Time evolution equation}

The time evolution equations of the scattering data are simple and uncoupled. For any $t \geq t_{0}$

$$
\begin{aligned}
a(k, t) & =a\left(k, t_{0}\right), \quad|k|=1, \\
b(k, t) & =b\left(k, t_{0}\right) \exp \left(\omega(k)\left(t-t_{0}\right)\right), \quad|k|=1, \\
\rho_{r}(t) & =\rho_{r}\left(t_{0}\right) \exp \left(\omega\left(k_{r}\right)\left(t-t_{0}\right)\right), \quad r=1, \ldots, R,
\end{aligned}
$$

where $\omega(k)=k-1 / k$. 


\subsection{Soliton}

The scattering data of a pure soliton are $\left(q_{0}>0, \epsilon_{0}= \pm 1\right)$ :

$$
\begin{aligned}
& a(k)=\epsilon_{0} \frac{k-k_{0}}{k k_{0}-1}, \quad b(k)=0, \quad k_{0}=\epsilon_{0} \exp \left(-q_{0}\right), \\
& \rho_{0}=\exp \left(2 q_{0} n_{0}(t)\right) \sinh \left(q_{0}\right), \quad n_{0}(t)=n_{0}(0)-\epsilon_{0} \sinh \left(q_{0}\right) / q_{0} t .
\end{aligned}
$$

The corresponding solution is:

$$
x_{n}(t)=-\ln \left[1+\frac{e^{q_{0}} \sinh \left(q_{0}\right)}{\cosh \left(q_{0}\left(n-n_{0}(t)\right)\right)} e^{-q_{0}\left(n-n_{0}(t)\right)}\right] .
$$

The soliton momentum and energy are:

$$
\mathcal{M}_{0}=2 \epsilon_{0} \sinh \left(q_{0}\right), \quad \mathcal{H}_{0}=2\left(\sinh \left(q_{0}\right) \cosh \left(q_{0}\right)-q_{0}\right) .
$$

Note that the soliton solution is negative-valued. Its velocity is negative (resp. positive) if the zero $k_{0}$ is positive (resp. negative). The Jost functions for the soliton are:

$$
\psi_{n}(k)=k^{n} A_{n}^{-1 / 2} \frac{1-k k_{0} A_{n}}{1-k k_{0}}, \quad \phi_{n}(k)=-\epsilon_{0} k^{-n} A_{n}^{-1 / 2} \frac{k-k_{0} A_{n}}{1-k k_{0}},
$$

where $A_{n}=\left(1+k_{0}^{2 n-2 n_{0}-2}\right) /\left(1+k_{0}^{2 n-2 n_{0}}\right)$.

\subsection{Conserved quantities}

Conserved quantities can be worked out as in any integrable system. The quantities

$$
I_{0}=-\frac{1}{2} \sum_{n=-\infty}^{\infty} \log \left(c_{n}\right), \quad I_{1}=-\sum_{n=-\infty}^{\infty} v_{n}, \quad I_{2}=\sum_{n=-\infty}^{\infty} 1-c_{n}-\frac{1}{2} v_{n}^{2}
$$

are three of the infinite number of conserved quantities for the homogeneous Toda chain. The first integral of motion is proportional to the total displacement $\lim _{n \rightarrow+\infty} x_{n}-\lim _{n \rightarrow-\infty} x_{n}=$ $-I_{0} / 2$. The second integral of motion is proportional to the momentum: $\sum_{n=-\infty}^{\infty} \dot{x}_{n}=-I_{1}$. Finally the Hamiltonian (2.2. grals of motion $\mathcal{H}_{0}=-I_{2}+2 I_{0}$. The derivation of the set of conserved quantities is based on the series expansion of the analytic function $a(k)$ as $k \rightarrow 0$ :

$$
\log a(k) \simeq \sum_{j=0}^{\infty} I_{j} k^{j}
$$

where the $I_{j}$ are time-independent. The conserved quantities can be expressed in terms of the scattering data. Defining $n(k):=-\log \left(1-\frac{|b|^{2}(k)}{|a|^{2}(k)}\right)$ for $k \in \mathbb{S}^{1}$, the integrals of motion $I_{j}$ can be decomposed into the sums of continuous and discrete parts:

$$
\begin{aligned}
& I_{0}=\frac{1}{4 i \pi} \oint_{\gamma_{u}} \frac{n(q)}{q} d q+\sum_{r=1}^{R} \log \left|k_{r}\right|, \\
& I_{j}=\frac{1}{2 i \pi} \oint_{\gamma_{u}} \frac{n(q)}{q^{j+1}} d q+\frac{1}{j} \sum_{r=1}^{R} k_{r}^{j}-k_{r}^{-j}, \quad j \geq 1 .
\end{aligned}
$$




\section{Propagation with an impure segment}

\subsection{Perturbation model}

We assume from now on that the masses of the particles are not equal:

$$
m_{n} \ddot{x}_{n}=\left(\exp \left(x_{n+1}-x_{n}\right)-\exp \left(x_{n}-x_{n-1}\right)\right),
$$

where $m_{n}$ is the mass of the particle at site $n$. A finite segment of impure masses is embedded into a homogeneous infinite chain:

$$
m_{n}= \begin{cases}1 & \text { for } n \leq 0 \text { and } n \geq N^{\varepsilon}+1, \\ 1+\varepsilon V_{n} & \text { for } 1 \leq n \leq N^{\varepsilon},\end{cases}
$$

where the small parameter $\varepsilon \in(0,1)$ characterizes the amplitude of the perturbation. $\left(V_{n}\right)_{n \in \mathbb{N}}$ is a chain of identically distributed random variables . They are zero-mean $\left\langle V_{n}\right\rangle=$ 0 ; they possess finite moments; the chain is stationary so $\left\langle V_{0} V_{n}\right\rangle=\left\langle V_{m} V_{m+n}\right\rangle$. We may think for instance at the discrete white noise, where the random variables $V_{n}$ are statistically independent $\left\langle V_{m} V_{n}\right\rangle=0$ if $m \neq n$ with the variance $\sigma^{2}=\left\langle V_{n}^{2}\right\rangle$. We may also consider a colored noise with a Gaussian autocorrelation function $\left\langle V_{0} V_{n}\right\rangle=\sigma^{2} \exp \left(-n^{2} / l_{c}^{2}\right)$ with variance $\sigma^{2}$ and correlation length $l_{c}$.

The length of the impure segment $N^{\varepsilon}$ is assumed to be large, of the order of $\varepsilon^{-2}$, and we set $N^{\varepsilon}=\left[l_{0} / \varepsilon^{2}\right]$. We introduce the slow variable $l$ as $n=\left[l / \varepsilon^{2}\right]$. Here the brackets stand for the integral part of a real number. We assume that a pure soliton is incoming from the left. The parameter of the soliton is $k_{0}=-e^{-q_{0}}$. Note that the total displacement, momentum and Hamiltonian are preserved:

$$
\begin{aligned}
\mathcal{D} & =\lim _{n \rightarrow+\infty} x_{n}-\lim _{n \rightarrow-\infty} x_{n}, \\
\mathcal{M} & =\sum_{n=-\infty}^{\infty} m_{n} \dot{x}_{n} \\
\mathcal{H} & =\sum_{n=-\infty}^{\infty} \frac{1}{2 m_{n}} \dot{x}_{n}^{2}+\left(\exp \left(x_{n}-x_{n-1}\right)-\left(x_{n}-x_{n-1}\right)-1\right) .
\end{aligned}
$$

\subsection{Evolution of the scattering data}

Let us consider a general form:

$$
\dot{c}_{n}=c_{n}\left(v_{n}-v_{n-1}\right)+\varepsilon R_{n}, \quad \dot{v}_{n}=c_{n+1}-c_{n}+\varepsilon S_{n} .
$$

We get the perturbation model $\left(\overline{3} \cdot \bar{l}^{\prime}\right)$ from this general form by setting $R_{n}=0$ and $S_{n}=$ $V_{n}\left(c_{n+1}-c_{n}\right)$. In such conditions the Jost coefficients satisfy the coupled equations:

$$
\begin{aligned}
& \frac{d a}{d t}=\varepsilon \frac{1}{k-k^{-1}}(\tilde{\gamma}(k) a+\gamma(k) b), \\
& \frac{d b}{d t}=\omega(k) b-\varepsilon \frac{1}{k-k^{-1}}\left(\gamma^{*}(k) a+\tilde{\gamma}(k) b\right),
\end{aligned}
$$


where

$$
\begin{aligned}
& \gamma(k)=\sum_{n} \psi_{n}(k)\left(\frac{R_{n}}{2 c_{n}^{1 / 2}} \psi_{n-1}(k)+\frac{R_{n+1}}{2 c_{n+1}^{1 / 2}} \psi_{n+1}(k)+S_{n} \psi_{n}(k)\right), \\
& \tilde{\gamma}(k)=\sum_{n} \psi_{n}^{*}(k)\left(\frac{R_{n}}{2 c_{n}^{1 / 2}} \psi_{n-1}(k)+\frac{R_{n+1}}{2 c_{n+1}^{1 / 2}} \psi_{n+1}(k)+S_{n} \psi_{n}(k)\right) .
\end{aligned}
$$

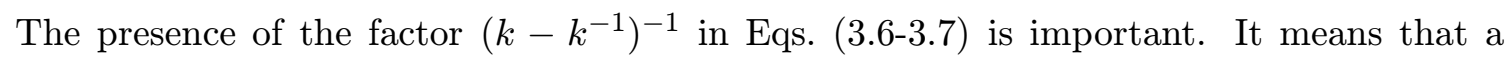
resonance exists close to the value $k=1$, and thus small solitons are likely to be generated, as was observed for the random Korteweg-de Vries (KdV) equation

The strategy we shall develop is based on the inverse scattering transform. The random perturbation induces variations of the spectral data. Calculating these changes we are able to find the effective evolution of the field and calculate the characteristic parameters of the wave. We are interested in the effective dynamics of the soliton propagating through large impure segments with length $N^{\varepsilon}=\left[l_{0} / \varepsilon^{2}\right]$. The total energy is conserved but the discrete and continuous components evolve during the propagation. The evolution of the continuous component corresponding to radiation will be found from the evolution equations of the Jost coefficients. The evolution of the soliton parameter will then be derived from the conservation of the total energy. However this approach turns out to be a little more tricky than expected because of the generation of new solitons.

\subsection{Convergence of the soliton parameter}

We can now state the main result of the paper. The proof follows closely the strategy developed in Ref. [i] the statement we need to define the concept of soliton gas in our framework. A soliton gas is a collection of solitons whose total energy goes to zero as $\varepsilon \rightarrow 0$ while the sum of their momenta is non-zero.

Proposition. The following event has a probability which goes to 1 as $\varepsilon \rightarrow 0$ : the wave scattered by a large impure segment with length $\left[l / \varepsilon^{2}\right]$ consists of one main soliton with parameter $q^{\varepsilon}(l)$, a soliton gas, and radiation. The process $\left(q^{\varepsilon}(l)\right)_{l \in\left[0, l_{0}\right]}$ converges in probability to the deterministic function $\left(q_{s}(l)\right)_{l \in\left[0, l_{0}\right]}$ which satisfies the ordinary differential equation:

$$
\frac{d q_{s}}{d l}=F\left(q_{s}\right)
$$

where

$$
F(q)=-\frac{1}{4 \pi} \int_{0}^{2 \pi} C^{2}(q, \theta) \hat{R}(2 K(q, \theta)) \frac{\sin ^{2}(\theta)}{\sinh ^{2}(q)} d \theta,
$$

$C^{2}(q, \theta)$ is the normalized energy density scattered by the soliton with parameter $q$ per unit distance for a discrete white noise:

$$
C(q, \theta)=\pi \frac{\sin \left(\theta-\frac{q \sin (\theta)}{\sinh (q)}\right)}{\sinh \left(\frac{\pi \sin (\theta)}{\sinh (q)}\right)},
$$


$\hat{R}(\kappa)$ is the discrete Fourier transform of the autocorrelation function of $\left(V_{n}\right)_{n \in \mathbb{N}}$

$$
\hat{R}(\kappa)=\sum_{n=-\infty}^{\infty}\langle V(0) V(n)\rangle \cos (\kappa n)
$$

$K(q, \theta)$ is the wavenumber

$$
K(q, \theta)=\theta-\frac{q \sin (\theta)}{\sinh (q)}
$$

The first assertion of the proposition means that the event "the transmitted wave consists of one soliton plus some other small amplitude wave" occurs with very high probability for small $\varepsilon$, while the second assertion gives the effective evolution equation of the parameter of the transmitted soliton in the asymptotic framework $\varepsilon \rightarrow 0$. Note that $\hat{R}$ is a positive realvalued (Wiener-Khintchine theorem). In case of a discrete white noise $\hat{R}(\kappa)$ is a constant equal to the variance $\sigma^{2}$.

The scattered wave consists of one main soliton (with parameter $q_{s}$ of order 1), a soliton gas (with quasi-zero energy but non-zero momentum), and radiation (associated with the continuous spectrum). The induced displacement, momentum and energy of the radiation are:

$$
\begin{aligned}
\mathcal{D}_{r} & =-\frac{1}{2 \pi} \int_{0}^{l} \int_{0}^{2 \pi} C^{2}\left(q_{s}(y), \theta\right) \hat{R}(2 K(q, \theta)) d \theta d y \\
\mathcal{M}_{r} & =\frac{1}{2 \pi} \int_{0}^{l} \int_{0}^{2 \pi} C^{2}\left(q_{s}(y), \theta\right) \hat{R}(2 K(q, \theta)) \cos (\theta) d \theta d y \\
\mathcal{H}_{r} & =\frac{1}{\pi} \int_{0}^{l} \int_{0}^{2 \pi} C^{2}\left(q_{s}(y), \theta\right) \hat{R}(2 K(q, \theta)) \sin ^{2}(\theta) d \theta d y
\end{aligned}
$$

respectively. We can also compute the induced displacement $\mathcal{D}_{g}$ and momentum $\mathcal{M}_{g}$ of the soliton gas since the total displacement and momentum are preserved by the perturbation, so:

$$
\begin{aligned}
\mathcal{D}_{g} & =2\left(q_{0}-q_{s}(l)\right)-\mathcal{D}_{r}, \\
\mathcal{M}_{g} & =-2\left(\sinh \left(q_{0}\right)-\sinh \left(q_{s}(l)\right)\right)-\mathcal{M}_{r} .
\end{aligned}
$$

The soliton gas actually consists of about $\varepsilon^{-2}$ solitons with parameters of order $q_{j}^{\varepsilon} \sim \varepsilon^{2}$. That is why the induced displacement (equal to $2 \sum_{j} q_{j}^{\varepsilon}$ ) and the momentum (equal to $2 \sum_{j} \varepsilon_{j} q_{j}^{\varepsilon}$ ) are of order 1 , while the energy (equal to $(4 / 3) \sum_{j} q_{j}^{\varepsilon 3}$ ) is of order $\varepsilon^{4}$ and hence asymptotically zero.

\subsection{Small soliton regime - white noise}

If $q \ll 1$, then the scattered energy density can be analyzed more precisely. It is found that the function $C$ is concentrated around $\theta=\pi$ with a bandwidth of the order of $q$ :

$$
C(q, \pi+q s) \stackrel{q \ll 1}{\simeq} \frac{2 \pi s q}{\sinh (\pi s)}
$$


This means that the radiation is going backward. Integrating establishes that $F$ is simply $F(q) \simeq-4 \sigma^{2} q^{3} / 15, q \ll 1$, and Eq. (3.10) can be solved $q_{s}(l)=q_{0} / \sqrt{1+8 \sigma^{2} q_{0}^{2} l / 15}$. In terms of energy, the decay rate reads as:

$$
\mathcal{H}_{s}(l)=\frac{\mathcal{H}_{0}}{\left(1+8 \sigma^{2} q_{0}^{2} l / 15\right)^{3 / 2}} .
$$

The small $q$ limit corresponds to the case of the broad discrete soliton. Then the continuum description is valid and the evolution of the relative displacements $u_{n}=x_{n}-x_{n-1}$ is described by the stochastically perturbed Boussinesq equation for the nonlinear string [i] $\left.{ }_{1} \overline{\bar{z}}\right]$

$$
b(x) u_{t t}=u_{x x}+u_{x} u_{x x}+\frac{1}{12} u_{x x x x},
$$

where $b(x)=1+\epsilon(x), \epsilon(x)$ is a white noise. The unperturbed Boussinesq equation is an integrable system, whose associated $L$ operator (in the Lax pair) has a complicated structure. The analysis of the soliton dynamics in the random nonlinear string has not performed yet. Our analysis predicts the $1 / l$ decay for the soliton amplitude propagating in the nonlinear string with the randomly distributed density.

Note finally that the decay rate as $l^{-3 / 2}$ for the soliton energy is in agreement with the numerical simulations carried out in $\left.\left[9_{1}^{1},\right]_{1}^{1} \overline{0}_{0}^{1}\right]$. However, as we shall now see this decay law is valid only for small-amplitude solitons.

\subsection{Large soliton regime - white noise}

The regime when $q_{0} \gg 1$ can also be analyzed precisely, while $q_{s}(l) \gg 1$. It is found that the function $C$ becomes independent of $\theta$ :

$$
C(q, \theta) \stackrel{q \gg 1}{\simeq} \frac{1}{2} e^{q} .
$$

This means that a broadband radiation is emitted. Integrating establishes that $F(q) \simeq-$ $\sigma^{2} / 4,, q \gg 1$, so that the decay rate of $q_{s}$ is linear $q_{s}(l)=q_{0}-\sigma^{2} l / 4$, which reads as an exponential decay in terms of the energy:

$$
\mathcal{H}_{s}(l)=\mathcal{H}_{0} \exp \left(-\frac{\sigma^{2} l}{2}\right)
$$

The energy decay rate is also independent of the energy of the incoming soliton. This remarkable feature was pointed out by the numerical simulations carried out in Ref. [i] $10-1]$ When the value $q_{s}$ becomes of the order of 1, the decay switches to the power law described in the previous section.

It should be noted that the decay rate of a large-amplitude soliton is higher than the one of a small amplitude soliton. This seems in contradiction with previous analysis of solitons driven by random perturbations for other types of integrable systems, such as the NLS


equation to our system as the discreteness plays a primary role when the soliton amplitude is large. The analysis of the randomly perturbed NLS equation had shown that nonlinearity 
may reduce the exponential localization. The proposed analysis of the randomly perturbed Toda system shows that the interplay between discreteness, nonlinearity and randomness is more complicated and may lead to an enhanced instability of a large-amplitude soliton. In the large-amplitude regime the soliton width is of the order of one site, and so is the correlation length of the discrete white noise. This involves a strong interaction between the fluctuations of the medium and the soliton.

\section{Conclusion}

We have studied the propagation of solitons through a segment of $N$ impure masses. We have shown that the decay rate of the soliton energy is an exponential decaying function of $N$ for large energies, with a decay rate that does not depend on the soliton. The scattering of a large-amplitude soliton is characterized by the emission of a broadband radiation in forward and backward directions. When the energy becomes small, the decay switches to the power law $N^{-3 / 2}$. The scattering of a small amplitude soliton is characterized by the emission of a narrowband backward-going radiation.

We have put into evidence that the scattering of the soliton generates not only continuous radiation, but also a soliton gas, that is to say a collection of $J^{\varepsilon}$, of order $\varepsilon^{-2}$, solitons with small parameters $q_{j}^{\varepsilon}$, of order $\varepsilon^{2}$ (remember $\varepsilon$ is the dimensionless parameter that governs the amplitude of the perturbation and the length of the segment $N=\left[l / \varepsilon^{2}\right]$ ). In the asymptotic framework where $\varepsilon$ goes to zero, the soliton gas has non-zero momentum, but zero energy. The production of the soliton gas is interesting by itself as a new phenomenon that is not encountered when a random NLS or AL equation is considered, but it should also be pointed out that this production is very important in that one cannot understand correctly the changes in the conservation equations without accounting for soliton production.

\section{Acknowledgments}

F.A. is grateful to Instituto de Fisica Teorica, UNESP for the hospitality and to FAPESP for the partial financial support.

\section{References}

[1] Nonlinearity with Disorder, Springer Proc. in Physics 67, F. Kh. Abdullaev, A. R. Bishop, and St. Pnevmatikos (Eds), Springer-Heidelberg 1992.

[2] Yu. S. Kivshar, S. A. Gredeskul, A. Sanchez, and L. Vazquez, Phys. Rev. Lett. 64 (1990) 1693.

[3] P. W. Anderson, Phys. Rev. 109 (1958) 1492.

[4] S.S. Abdullaev, F.Kh. Abdullaev, Izv. VUZ'0v - Radiofizika 23 (1980) 766. (In Russian).

[5] J.D. Maynard, Rev.Mod.Phys. 73 (2001) 401.

[6] J. Garnier, SIAM J. Appl. Math. 58 (1998) 1969. 
[7] Q. Li, C.M. Soukoulis, St. Pnevmatikos, and E. N. Economou, Phys. Rev. B 38 (1988) 11888.

[8] G. S. Zavt, M. Wagner, and A. Lutze, Phys. Rev. E 47 (1993) 4108.

[9] S. Ishiwata, Y. Okada, S. Watanabe, and H. Tanaka, J. Phys. Soc. Jpn. 59 (1990) 3029.

[10] Y. Kubota and T. Odagaki, Phys.Rev. E 61 (2000) 3133.

[11] T. Ilzuka, T. Nakao, and M. Wadati, Phys. Soc. Jpn. 60 (1991) 4167.

[12] F.Kh. Abdullaev, Theory of solitons in inhomogeneous media, Wiley Chichester 1994.

[13] J. Garnier, Phys.Rev. E 63 (2001) 026608.

[14] V. Muto, P. S. Lomdahl, and P. L. Christiansen, Phys.Rev. A 42 (1990) 7452.

[15] M. Toda, Theory of nonlinear lattices, Springer Heidelberg 1981.

[16] V. S. Gerdjikov, D. J. Kaup, I. M. Uzunov, and E. G. Evstatiev, Phys. Rev. Lett. 77 (1996) 3943.

[17] S. V. Manakov, S. Novikov, J. P. Pitaevskii, and V. E. Zakharov, Theory of solitons, Consultants Bureau New York 1984.

[18] J. Garnier, J. Statist. Phys. 105 (2001) 789.

[19] V. I. Karpman, Phys. Scr. 20 (1979) 462. 